\title{
Psychometric evaluation of Azeri version of the head and neck cancer specific quality of life questionnaire (EORTC QLQ-H\&N43)
}

Mahammad M. Davudov ${ }^{1,2}$, Chingiz Rahimov², Iraj Harirchi ${ }^{1}$, Zoheir Mirzajani ${ }^{1,2}$, Namig Amiraliyev ${ }^{3}$, Kanan Amiraliyev ${ }^{2}$, Narmin Rustamovaa ${ }^{2}$ Jayran Zebardast ${ }^{1}$ and Ali Montazeri ${ }^{4,5^{*}}$ (D)

\begin{abstract}
Background: Oral cancer surgery can have a deep effect on the quality of life in the patient both in terms of functional and psychological aspects. This study aimed to translate and validate the European Organization for Research and Treatment of Cancer head and neck cancer specific quality of life questionnaire (EORTC QLQ-H\&N43) in Azerbaijan.
\end{abstract}

Methods: Forward-backward translation was applied in order to translate the EORTC QLQ-H\&N43 from English into Azeri. Then, a sample of patients with oral cancer attending a teaching hospital affiliated to Azerbaijan Medical University completed the EORTC QLQ-C30 (the core cancer specific questionnaire), and the EORTC QLQ-H\&N43. To evaluate psychometric properties of the QLQ-H\&N43, known groups validity, convergent and divergent validity was performed. Internal consistency reliability was examined by estimating the Crornbach's alpha coefficient.

Results: Ninety-six patients with confirmed diagnosis of oral cancer were entered into the study. The mean age of patients was $59.6(S D=10.7)$ years and 36 patients (37.5\%) diagnosed as having stage IV and 10 patients (10.5\%) were metastatic. The results obtained from comparing quality of life scores among these patients showed that the questionnaire was able to differentiate among patients who differed in stage and metastasis lending support to its validity. In addition convergent and divergent validity showed satisfactory results. The internal consistency of the multi-item scales as assessed by the Cronbach's alpha coefficient showed acceptable results (alpha ranging from 0.66 to 0.78 ).

Conclusion: The findings suggest that in general the Azeri version of EORTC QLQ-H\&N43 has satisfactory internal consistency reliability and validity, but additional psychometric evaluation is needed to draw firm conclusions.

Keywords: Psychometric analysis, EORTC QLQ-H\&N43, Azerbaijan, Oral cancer

\footnotetext{
* Correspondence: montazeri@acecr.ac.ir

${ }^{4}$ Population Health Research Group, Health Metrics Research Center, Iranian Institute for Health Sciences Research, ACECR, Tehran, Iran

${ }^{5}$ Faculty of Humanity Sciences, University of Science and Culture, ACECR, Tehran, Iran

Full list of author information is available at the end of the article
}

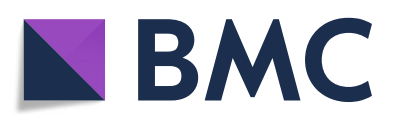

() The Author(s). 2020 Open Access This article is licensed under a Creative Commons Attribution 4.0 International License, which permits use, sharing, adaptation, distribution and reproduction in any medium or format, as long as you give appropriate credit to the original author(s) and the source, provide a link to the Creative Commons licence, and indicate if changes were made. The images or other third party material in this article are included in the article's Creative Commons licence, unless indicated otherwise in a credit line to the material. If material is not included in the article's Creative Commons licence and your intended use is not permitted by statutory regulation or exceeds the permitted use, you will need to obtain permission directly from the copyright holder. To view a copy of this licence, visit http://creativecommons.org/licenses/by/4.0/ The Creative Commons Public Domain Dedication waiver (http://creativecommons.org/publicdomain/zero/1.0/) applies to the data made available in this article, unless otherwise stated in a credit line to the data. 


\section{Background}

Oral cancer and its treatment have substantial effect on patients' quality of life [1]. More importantly there are several factors that deepen this effect. For instance patients with oral cancer who receive surgery finds problems with appearance, speech, ability to breathe, eat and swallow [2]. All these could have both short- and longterm effect on patients' physical, mental and social wellbeing [3]. Thus in clinical settings it is important to assess health-related quality of life in this population in order to evaluate treatment outcomes. As such questionnaires that have been developed by the European Organization for Research and Treatment of Cancer (EORTC) are very well known and are frequently used in outcome studies in oncology. For instance the EORTC QLQ-C30 was developed for measuring quality of life in all cancer patients (core cancer questionnaire) [4] and consequently for many anatomical sites specific instruments proposed to be used in conjunction with core questionnaire and currently they are in use worldwide. One of these instruments is the specific questionnaire that was developed for measuring quality of life in patients with head and neck cancer. The questionnaire first introduced with 35 items in 1999 and was named the EORTC QLQ-H\&N35 [5, 6]. Since then the QLQH\&N35 was translated and validated in different countries and a number of various languages including US English [7], Italian [8] Taiwan Chinese [9], Japanese [10], German [11], Cantonese (Hong Kong) [12], Greek [13], Chinese [14], Mexican Spanish [15], and Arabic [16].

However later on after comprehensive review by the EORTC Quality of Life Group and the EORTC Head and Neck Cancer Group it was updated and it was tested in Danish, Dutch, French, German, Greek, Hebrew, Italian, Japanese, Mandarin, Norwegian, Polish, Portuguese, Spanish, and Swedish following forward-backward translation of original English version of the questionnaire. This update version of the questionnaire contains 43 items and examines several important symptoms in head and neck cancer patients [17].

Since the Azeri version of the questionnaire was not available we aimed to translate and validate the QLQH\&N43 questionnaire in Azerbaijan.

\section{Methods}

\section{QLQ-H\&N43}

The EORTC QLQ-H\&N43 is a supplementary module for the EORTC QLQ-C30 that measures quality of life in patients with had and neck cancers. It contains 43 items tapping into 6 multi-item and 13 single-item symptom subscales namely pain, swallowing, senses problems, speech problems, trouble with social eating, less sexuality, teeth, dry mouth/sticky saliva, body image, shoulder pain, skin problems, anxiety, trouble with social contact, opening mouth, coughing, lymphedema, problems wound healing, weight loss, neurological problems [17]. Each item is rated on a 4-point Likert scale and scores for each subscale ranges from 0 to 100 where higher scores indicate greater symptoms.

\section{Translation}

After asking permission from the EORTC Quality of Life Study Group, forward-backward procedure was applied to translate the questionnaires from English into Azeri. Two independent translators translated the original questionnaire into Azeri. Although there were some differences between two translations, the research team dealt with these differences and provided a single forward version of the questionnaire. For instance there were differences in translating skin 'rash' or 'skin changed color'. Consequently two other bilingual translators back translated the questionnaires from Azeri into English. Accordingly a single back translated version of the questionnaire made available and it was checked with the original questionnaire for any errors or deviations. To check cultural relevance of the translation, and ease of comprehension five patients (not included in the current study) the instrument was pre-tested. However, since it was verified, the provisional version of the Azeri questionnaire was subjected to psychometric properties.

\section{Psychometric evaluation}

A cross sectional study was conducted on a sample of patients with confirmed oral cancer attending a teaching hospital affiliated to Medical University of Azerbaijan in Baku from year 2011 to 2017. They all completed the study questionnaires including the Azeri version of the EORTC QLQ-C30 and the EORTC QLQ-H\&N43.

\section{Statistical analysis}

Validity was examined using known groups comparison. We hypothesized that the questionnaire should be able to differentiate between patients who are differing in stage and metastasis. In fact we hypothesized that patients with lower stage and no metastasis should score better than patients with higher stage and having metastasis. For comparison the Mann-Whitney and KrurskalWallis tests were used. In addition convergent and divergent validity (discriminant validity) was examined by estimating correlation coefficient between the EORTC QLQ-C30 and the EORTC QLQ-H\&N43 scores. Due to skewed distribution of the data we used the Spearman's rho coefficient. Correlation coefficients ranging 0.1-0.3 were considered low, $0.31-0.5$ as moderate, and those exceeding 0.5 as high. Internal consistency reliability for multi-items subscales (having at least 3 items) was assessed using the Cronbach's alpha coefficient. Alpha 
Table 1 Demographic and clinical characteristics of the study sample $(n=96)$

\begin{tabular}{|c|c|c|}
\hline & Frequency & Percent \\
\hline \multicolumn{3}{|l|}{ Gender } \\
\hline Male & 67 & 69.8 \\
\hline Female & 29 & 30.2 \\
\hline \multicolumn{3}{|l|}{ Age } \\
\hline$<60$ & 49 & 51 \\
\hline$\geq 60$ & 47 & 49 \\
\hline \multicolumn{3}{|c|}{ Tumor grade } \\
\hline 1 & 16 & 16.7 \\
\hline 2 & 27 & 28.1 \\
\hline 3 & 30 & 31.2 \\
\hline 4 & 23 & 24 \\
\hline \multicolumn{3}{|l|}{ Nodes } \\
\hline 0 & 65 & 68 \\
\hline 1 & 13 & 13.5 \\
\hline 2 & 11 & 11.5 \\
\hline 3 & 7 & 7.3 \\
\hline \multicolumn{3}{|c|}{ Metastasis } \\
\hline No & 86 & 89.6 \\
\hline Yes & 10 & 10.4 \\
\hline \multicolumn{3}{|c|}{ Radiotherapy } \\
\hline No & 56 & 58.3 \\
\hline Yes & 40 & 41.7 \\
\hline \multicolumn{3}{|l|}{ Stage } \\
\hline$|\&| \mid$ & 34 & 35.4 \\
\hline III & 26 & 27.1 \\
\hline IV & 36 & 37.5 \\
\hline \multicolumn{3}{|c|}{ Chemotherapy } \\
\hline No & 75 & 78.1 \\
\hline Yes & 21 & 21.9 \\
\hline
\end{tabular}

coefficient equal or greater than 0.7 was thought satisfactory.

\section{Results}

A total of 96 patients (67 men and 29 women) who underwent flap reconstruction for oral cancer in Azerbaijan were studied. The mean age of patients was $59.6(\mathrm{SD}=10.7)$ years ranging from 30 to 82 . In 47 cases, age was lower than 60 years. The characteristics of patients are given in Table 1 . In addition the descriptive quality of life scores for all patients including the floor and ceiling effects are presented in Table 2.

\section{Validity of the QOL-H\&N43}

\section{Known groups validity}

As shown in Tables 3 and 4 although not significant, the QLQ-H\&N43 differentiated patients who differed in stage and metastasis. As expected patients with the advanced disease scored higher almost on all symptoms and thus lending support to the validity of the questionnaire.

\section{Convergent and divergent validity (discriminant validity)}

The correlation between the EORTC QLQ-C30 and the EORTC QLQ-H\&N43 scores was examined. As expected almost in all instances relevant subscales showed acceptable correlations and those not related exhibited low correlations. The detailed results are shown in Table 5.

\section{Internal consistency reliability}

The Cronbach's alpha coefficient for the multi-item scales of the QLQ-H\&N43 ranged from 0.66 to 0.78 indicating that the internal consistency of the Azeri version of the questionnaire was acceptable. The results are shown in Table 6.

\section{Discussion}

The findings from this study indicated that the Azeri version of the EORTC QLQ-H\&N43 is a valid instrument for measuring quality of life in head and neck cancer patients. The internal consistency in five out of seven multi-item subscales was good and only the Cronbach's alpha for social eating (0.66) and body image (0.69) were slightly lower than acceptable threshold (0.7). Perhaps these could be due to cultural differences that exist among different nations. For instance, social eating is less common in Azerbaijan.

The authors of the original study that introduced the EORTC QLQ-H\&N43 indicated that they analyzed the data in accordance with the EORTC Quality of Life Group QLG Module Development Guidelines. Thus, to retain an item in the module, they used 8 predefined criteria such as relevance, floor and ceiling effects, item difficulty, and compliance. They also used additional measures including the Cronbach's alpha coefficient to assess internal consistency for multi-item subscales. The Cronbach's alpha for hypothesized subscales they included in the module ranged from 0.77 to 0.87 that were well above acceptable values [17].

To the best of our knowledge contrary to expectation other than the original study, the current study is the second independent one that reports on psychometric properties of the QLQ-H\&N43. The Serbian version of the QLQ-H\&N43 was the first study that reported on psychometric evaluation of the questionnaire. The study included 170 patients and found good internal consistency for 5 out of 7 multi-item subscales. The study also showed that the questionnaire had acceptable validly (known groups validity) where patients who differed in type of laryngectomy, adjuvant therapy or 5-year 
Table 2 The EORTC QLQ-C30 and the QOL-H\&N43 scores for the patients $(n=96)$

\begin{tabular}{|c|c|c|c|}
\hline & Mean & Standard deviation & Floor/ceiling effects (\%) \\
\hline \multicolumn{4}{|l|}{ QLQ-C30 } \\
\hline \multicolumn{4}{|l|}{ Functioning $^{a}$} \\
\hline Physical functioning & 90.5 & 13.7 & $0.0 / 12.7$ \\
\hline Role functioning & 83.8 & 16.8 & $0.0 / 15.4$ \\
\hline Emotional functioning & 72.1 & 17.0 & $0.0 / 5.4$ \\
\hline Cognitive functioning & 86.5 & 20.2 & $0.0 / 9.7$ \\
\hline Social functioning & 80.7 & 20.5 & $0.0 / 12.8$ \\
\hline Global quality of life & 61.7 & 14.4 & $1.1 / 12.0$ \\
\hline \multicolumn{4}{|l|}{ Symptoms ${ }^{b}$} \\
\hline Fatigue & 27.1 & 22.6 & $11.0 / 2.2$ \\
\hline Nausea and vomiting & 8.51 & 17.2 & $3.3 / 13.0$ \\
\hline Pain & 33.3 & 19.2 & $7.6 / / 9.8$ \\
\hline Dyspnoea & 16.6 & 22.3 & $14.4 / 10.8$ \\
\hline Insomnia & 12.3 & 23.5 & $8.6 / 1.1$ \\
\hline Appetite loss & 18.1 & 24.9 & $8.0 / 15.2$ \\
\hline Constipation & 34.0 & 30.4 & $3.7 / 6.5$ \\
\hline Diarrhoea & 23.9 & 28.0 & $8.9 / 4.3$ \\
\hline Financial difficulties & 37.6 & 26.7 & $1.1 / 25.0$ \\
\hline \multicolumn{4}{|l|}{ QLQ-H\&N43 ${ }^{b}$} \\
\hline HN Pain & 29.5 & 22.4 & $9.9 / 1.1$ \\
\hline HN Swallowing & 29.6 & 16.4 & $4.3 / 5.4$ \\
\hline HN Senses problems & 15.0 & 18.3 & $6.5 / 2.2$ \\
\hline HN Speech problems & 26.3 & 16.7 & $5.4 / 1.1$ \\
\hline HN Trouble with social eating & 28.0 & 18.2 & 10.9/0.0 \\
\hline HN Less sexuality & 21.4 & 27.3 & $4.9 / 2.2$ \\
\hline HN Problems with teeth & 44.5 & 25.1 & $13.0 / 5.4$ \\
\hline HN Dry mouth/Sticky saliva & 11.0 & 19.8 & $8.5 / 1.1$ \\
\hline HN Body image & 24.8 & 21.1 & $13.9 / 0.0$ \\
\hline HN Shoulder pain & 5.49 & 15.5 & $7.3 / 0.0$ \\
\hline HN Skin problems & 7.65 & 17.0 & $12.0 / 1.1$ \\
\hline HN Anxiety & 48.3 & 30.1 & $17.8 / 5.7$ \\
\hline HN Trouble with social contact & 12.4 & 24.6 & $6.6 / 2.2$ \\
\hline HN Opening mouth & 31.5 & 32.1 & $13.4 / 6.5$ \\
\hline HN Coughing & 11.2 & 23.3 & $14.1 / 2.8$ \\
\hline HN Lymphedema & 9.52 & 18.7 & 15.8/0.0 \\
\hline HN Weight loss & 27.0 & 31.5 & $14.4 / 1.1$ \\
\hline HN Problems wounds healing & 12.9 & 21.6 & $12.1 / 4.4$ \\
\hline HN Neurological problems & 11.1 & 21.1 & $13.8 / 2.2$ \\
\hline
\end{tabular}

${ }^{\text {aHigher scores indicate better conditions }}$

${ }^{\mathrm{b}}$ Higher scores indicate greater symptoms

survival scored differently in the expected directions [18].

We used known groups comparison for the validity purpose. As expected the Azeri version of the questionnaire well differentiated among patients who differed in stage and metastasis, although in most instances the differences among patients were not significant. For instance as reported in Table 5, only 8 out of 19 subscale showed statistically significant difference between the two groups, and in Table 6, only 2 out of 19 subscale 
Table 3 The QLQ-H\&N43 scores in patients with and without metastasis*

\begin{tabular}{llll}
\hline & Patients without metastasis $(\boldsymbol{n}=86)$ & Patients with metastasis $(\boldsymbol{n}=10)$ & Mean (SD) \\
\hline Pain & Mean (SD) & $35.1(30.5)$ & $38.8(13.1)$ \\
Swallowing & $28.9(21.5)$ & $25.9(23.7)$ & 0.66 \\
Senses problems & $28.6(16.5)$ & $44.4(12.4)$ & 0.06 \\
Speech problems & $13.8(17.4)$ & $35.1(24.5)$ & 0.10 \\
Trouble with social eating & $24.4(16.0)$ & $35.1(31.6)$ \\
Less sexuality & $27.3(17.4)$ & $61.7(13.7)$ \\
Problems with teeth & $19.9(26.6)$ & $16.6(20.4)$ \\
Dry mouth/Sticky saliva & $42.6(25.4)$ & $25.9(21.5)$ \\
Body image & $10.4(19.7)$ & $12.9(23.2)$ \\
Shoulder pain & $24.7(21.2)$ & $25.9(35.1)$ \\
Skin problems & $4.67(14.4)$ & $53.7(30.9)$ & 0.001 \\
Anxiety & $5.62(12.5)$ & $37.0(48.8)$ \\
Trouble with social contact & $47.7(30.2)$ & $59.2(27.7)$ & 0.25 \\
Opening mouth & $9.7(21.2)$ & $33.3(44.0)$ & 0.09 \\
Coughing & $28.5(31.2)$ & $25.9(27.7)$ & 0.02 \\
Lymphedema & $8.8(18.8)$ & $44.4(28.8)$ & 0.29 \\
Weight loss & $7.7(16.8)$ & $25.9(14.6)$ & 0.76 \\
Problems wounds healing & $25.1(31.4)$ & $22.2(23.5)$ & 0.10 \\
Neurological problems & $11.5(21.8)$ & & 0.07 \\
\hline
\end{tabular}

*Higher scores indicate greater symptoms

**Derived from Mann-Whitney U test

Table 4 The QLQ-H\&N43 scores by stage of the disease*

\begin{tabular}{|c|c|c|c|c|}
\hline & Stage | \& || $(\boldsymbol{n}=34)$ & Stage III $(\boldsymbol{n}=26)$ & Stage IV $(\boldsymbol{n}=36)$ & \\
\hline & Mean (SD) & Mean (SD) & Mean (SD) & $P^{* *}$ \\
\hline Pain & $26.4(23.5)$ & $28.8(15.3)$ & $33.6(26.1)$ & 0.46 \\
\hline Swallowing & $29.4(15.7)$ & $29.4(19.4)$ & $29.9(14.9)$ & 0.98 \\
\hline Senses problems & $11.5(16.1)$ & $14.7(20.4)$ & $18.2(17.6)$ & 0.27 \\
\hline Speech problems & $20.7(17.1)$ & $25.1(15.5)$ & $33.3(15.3)$ & 0.006 \\
\hline Trouble with social eating & $23.0(18.3)$ & $26.4(17.5)$ & $33.8(17.9)$ & 0.09 \\
\hline Less sexuality & $16.6(26.0)$ & $23.9(28.6)$ & $24.3(27.5)$ & 0.38 \\
\hline Problems with teeth & $36.0(23.9)$ & $41.8(25.7)$ & $55.9(22.1)$ & 0.007 \\
\hline Dry mouth/Sticky saliva & $9.3(18.4)$ & $10.9(18.2)$ & $13.4(23.5)$ & 0.68 \\
\hline Body image & $19.0(18.9)$ & $26.7(20.8)$ & $29.4(23.0)$ & 0.11 \\
\hline Shoulder pain & $3.8(13.5)$ & $5.5(15.4)$ & $6.7(17.3)$ & 0.74 \\
\hline Skin problems & $4.7(12.6)$ & $6.3(13.8)$ & $11.4(22.3)$ & 033 \\
\hline Anxiety & $43.4(31.7)$ & $47.4(30.8)$ & $54.3(27.8)$ & 0.39 \\
\hline Trouble with social contact & $8.1(14.5)$ & $11.5(24.8)$ & 17.7 (31.6) & 0.62 \\
\hline Opening mouth & $21.5(27.0)$ & $37.1(39.2)$ & $37.5(29.0)$ & 0.08 \\
\hline Coughing & $08.9(17.7)$ & $10.7(21.2)$ & $13.5(29.1)$ & 0.99 \\
\hline Lymphedema & $03.8(14.3)$ & $11.1(19.8)$ & $12.5(20.3)$ & 0.10 \\
\hline Weight loss & $20.2(32.2)$ & $24.3(30.6)$ & $36.5(30.2)$ & 0.05 \\
\hline Problems wounds healing & $5.1(12.2)$ & $13.9(20.6)$ & $18.1(26.4)$ & 0.08 \\
\hline Neurological problems & $6.4(16.3)$ & $8.0(16.7)$ & $18.2(26.9)$ & 0.08 \\
\hline
\end{tabular}


Table 5 Correlation between the EORTC QLQ-C30 functioning and global quality of life and the EORTC QLQ-H\&N43 scores*

\begin{tabular}{lllllll}
\hline & PF & RF & EF & CF & SF & GQOL \\
\hline Pain & $-0.38(0.004)$ & $-0.33(0.007)$ & $-0.42(<0.001)$ & $-0.14(0.18)$ & $-0.20(0.68)$ & $-0.32(0.002)$ \\
Swallowing & $-.0 .11(0.29)$ & $-.012(0.27)$ & $-0.29(0.005)$ & $-0.15(0.15)$ & $-.0 .25(0.02)$ & $-0.34(0.01)$ \\
Senses problems & $-0.28(0.006)$ & $-0.18(0.07)$ & $-0.37(<0.001)$ & $-0.22(0.03)$ & $-0.26(0.01)$ & $-0.17(0.09)$ \\
Speech problems & $-0.36(<0.001)$ & $-0.16(0.12)$ & $-0.19(0.06)$ & $-0.15(0.16)$ & $-0.45(<0.001)$ & $-0.43(<0.001)$ \\
Trouble with social eating & $-0.20(0.05)$ & $-0.25(0.01)$ & $-0.37(<0.001)$ & $-0.14(0.19)$ & $-0.37(<0.001)$ & $-0.23(0.02)$ \\
Less sexuality & $-0.53(<0.001)$ & $-0.35(0.001)$ & $-0.24(0.02)$ & $-0.46(<0.001)$ & $-0.17(0.11)$ & $-0.40(<0.001)$ \\
Problems with teeth & $-0.50(<0.001)$ & $-0.24(0.02)$ & $-0.26(0.01)$ & $-0.04(0.71)$ & $-0.27(0.009)$ & $-0.37(<0.001)$ \\
Dry mouth/Sticky saliva & $-0.29(0.006)$ & $-0.15(0.16)$ & $-0.14(0.18)$ & $-0.17(0.11)$ & $-0.09(0.37)$ & $-0.05(0.62)$ \\
Body image & $-0.07(0.48)$ & $-0.01(0.92)$ & $-0.24(0.03)$ & $-0.18(0.37)$ & $-0.22(0.03)$ & $-0.55(<0.001)$ \\
Shoulder pain & $-0.30(0.003)$ & $-0.51(<0.001)$ & $-0.15(0.14)$ & $-0.15(0.14)$ & $-0.14(0.16)$ & $-0.41(<0.001)$ \\
Skin problems & $-0.08(0.43)$ & $-0.18(0.08)$ & $-0.05(0.60)$ & $-0.04(0.64)$ & $-0.18(0.09)$ & $-0.21(0.05)$ \\
Anxiety & $-0.27(0.01)$ & $-0.04(0.69)$ & $-0.80(<0.001)$ & $-0.04(0.70)$ & $-0.05(0.63)$ & $-0.29(0.006)$ \\
Trouble with social contact & $-0.32(0.002)$ & $-0.16(0.11)$ & $-0.10(0.32)$ & $-0.23(0.03)$ & $-0.38(<0.001)$ & $-0.31(0.004)$ \\
Opening mouth & $-0.32(0.004)$ & $-0.37(<0.001)$ & $0.34(<0.001)$ & $-0.24(0.02)$ & $-0.21(0.04)$ & $-0.51(<0.001)$ \\
Coughing & $-0.29(0.005)$ & $-0.02(0.88$ & $-0.01(0.93)$ & $-0.13(0.21)$ & $-0.02(0.8)$ & $-0.13(0.22)$ \\
Lymphedema & $-0.30(0.003)$ & $-0.15(0.14)$ & $-0.15(0.14)$ & $-0.15(0.14)$ & $-0.15(0.14)$ & $-0.10(0.33)$ \\
Weight loss & $-0.25(0.01)$ & $-0.13(0.22)$ & $-0.14(0.16)$ & $-0.13(0.22)$ & $-0.24(0.02)$ & $-0.43(<0.001)$ \\
Problems wounds healing & $-0.38(<0.001)$ & $-0.23(0.03)$ & $-0.28(0.007)$ & $-0.25(0.01)$ & $-0.41(<0.001)$ & $-0.43(<0.001)$ \\
Neurological problems & $-0.35(0.001)$ & $-0.40(<0.001)$ & $-0.27(0.01)$ & $-0.33(0.002)$ & $-0.35(0.001)$ & $-0.31(0.003)$ \\
\hline
\end{tabular}

*Figures are Spearman's rho coefficients and ( $P$ values). Negative signs are due to directions of scoring where higher scores on the EORTC QLQ-C30 represent better conditions and on the EORTC QLQ-H\&N43 indicate greater symptoms. Abbreviations read as follows: PF physical functioning, RF role functioning, CF cognitive functioning, SF social functioning, GQOL global quality of life

showed statistically significant difference. One explanation for such observation might be related to the small sample size in each group. In addition since we analyzed the data for all types of oral cancers, therefore one might argue that if there was an opportunity to analyze the data for each sub-types of oral cancer, then it was possible to obtain significant results for all measures assessed. Finally as pointed out by Polit and Yang when performing known groups validity the direction of differences for scores among sub-groups (as hypothesized) are more important than statistical significant level values [19].

Table 6 Cronbach's a for the EORTC QLQ-H\&N43 multi-item subscales

\begin{tabular}{lll}
\hline & Number of items & Cronbach's a \\
\hline Pain & 4 & 0.78 \\
Swallowing & 4 & 0.70 \\
Speech problems & 5 & 0.72 \\
Social eating & 4 & 0.66 \\
Problems with teeth & 3 & 0.73 \\
Body image & 3 & 0.69 \\
Skin problems & 3 & 0.72 \\
\hline
\end{tabular}

Interestingly the authors of the QLQ-H\&N43 stated that the QLQ-H\&N35 could still be used in ongoing or future studies if the investigators prefer to use this head and neck module version. However, they believe in studies investigating multimodal treatment or targeted therapies, the QLQ-H\&N43 might be more suitable to detect differences between patient groups [17].

This study has some limitations. The sample size was relatively small. Secondly we did not perform test-retest analysis to investigate the stability. It seems that for using the Azeri version of the QLQ-H\&N43 in future outcome studies we still need to perform further psychometric evaluations. However, one should note that the QLQ-H\&N43 should be used with conjunction of the EORTC QLQ-C30 core cancer questionnaire, which is now available in Azeri version, too [20].

\section{Conclusion}

The findings of this study suggest that in general the Azeri version of EORTC QLQ-H\&N43 has satisfactory internal consistency reliability and validity, but additional psychometric evaluation is needed to draw firm conclusions.

\section{Abbreviations}

EORTC QLQ-C30: The European Organization for Research and Treatment of Cancer core quality of life questionnaire; EORTC QLQ-H\&N35: The European 
Organization for Research and Treatment of Cancer head and neck cancer specific quality of life questionnaire- 35 items; EORTC QLQ-H\&N43: The European Organization for Research and Treatment of Cancer head and neck cancer specific quality of life questionnaire-43 items

\section{Acknowledgments}

We are grateful to all patients who participated in the study. We are also grateful to all academic staff in oral and maxillofacial surgery department and oncology department of Azerbaijan Medical University for help and support.

\section{Disclosure of results before publication}

The results have not been presented or published before.

\section{Authors' contributions}

MMD and ZM were the main investigators. KA and NR collected the data and wrote the first draft. $I H$ and $C R$ supervised the study. NA operated patients and contributed to study design. JZ contributed to data entry and data analysis. AM supervised the study and contributed to analysis and provided the final draft. All authors read and approved the manuscript.

\section{Funding}

Azerbaijan Medical University and Cancer Institute of Tehran University of Medical Sciences supported the study.

\section{Availability of data and materials}

All data presented in this paper are available from the corresponding authors on a reasonable request.

\section{Ethics approval and consent to participate}

The ethics committee of Tehran University of Medical Sciences approved the study. All patients complete written informed consent before the study commence.

\section{Consent for publication}

Not applicable.

\section{Competing interests}

The authors declare that they have no competing interests.

\section{Author details}

${ }^{1}$ Cancer Institute, Tehran University of Medical Sciences, Tehran, Iran. ${ }^{2}$ Department of Oral and Maxillofacial Surgery, Azerbaijan Medical University, Baku, Azerbaijan. ${ }^{3}$ Department of Oncology, Azerbaijan Medical University, Baku, Azerbaijan. ${ }^{4}$ Population Health Research Group, Health Metrics Research Center, Iranian Institute for Health Sciences Research, ACECR, Tehran, Iran. ${ }^{5}$ Faculty of Humanity Sciences, University of Science and Culture, ACECR, Tehran, Iran.

Received: 15 April 2019 Accepted: 20 July 2020

Published online: 23 July 2020

\section{References}

1. Parkar SM, Shah MN. A relationship between quality-of-life and head and neck cancer: a systemic review. South Asian J Cancer. 2015;4(4):179-82.

2. Høxbroe Michaelsen S, Grønhøj C, Høxbroe Michaelsen J, Friborg J, von Buchwald C. Quality of life in survivors of oropharyngeal cancer: a systematic review and meta-analysis of 1366 patients. Eur J Cancer. 2017;78: 91-102.

3. Clasen D, Keszte J, Dietz A, Oeken J, Meister EF, Guntinas-Lichius O, Pabst F, Büntzel J, Jenzewski EM, Singer S, Meyer A. Quality of life during the first year after partial laryngectomy: longitudinal study. Head Neck. 2018;40(6): 1185-95.

4. Aaronson NK, Ahmedzai S, Bergman B, Bullinger M, Cull A, Duez NJ, Filiberti A, Flechtner H, Fleishman SB, de Haes JC, Kaasa S, Klee M, Osoba D, Razavi D, Rofe P, Schraub S, Sneeuw K, Sullivan M, Takeda F. The European Organization for Research and Treatment of Cancer QLQ-C30: a quality-oflife instrument for use in international clinical trials in oncology. J Natl Cancer Inst. 1993:85(5):365-76.

5. Bjordal K, Ahlner-Elmqvist M, Tollesson E, Jensen AB, Razavi D, Maher EJ Kaasa S. Development of a European Organization for Research and
Treatment of Cancer (EORTC) questionnaire module to be used in quality of life assessments in head and neck cancer patients. EORTC quality of life study group. Acta Oncol. 1994;33:879-85.

6. Bjordal K, Hammerlid E, Ahlner-Elmqvist M, de Graeff A, Boysen M, Evensen JF, Biörklund A, de Leeuw JR, Fayers PM, Jannert M, Westin T, Kaasa S. Quality of life in head and neck cancer patients: validation of the European Organization for Research and Treatment of Cancer quality of life questionnaire-H\&N35. J Clin Oncol. 1999;17(3):1008-19.

7. Sherman AC, Simonton S, Adams DC, Vural E, Owens B, Hanna E. Assessing quality of life in patients with head and neck cancer: cross-validation of the European Organization for Research and Treatment of Cancer (EORTC) quality of life head and neck module (QLQ-H\&N35). Arch Otolaryngol Head Neck Surg. 2000;126(4):459-67.

8. Zotti P, Lugli D, Vaccher E, Vidotto G, Franchin G, Barzan L. The EORTC quality of life questionnaire-head and neck 35 in Italian laryngectomized patients. Qual Life Res. 2000;9(10):1147-53.

9. Chie WC, Hong RL, Lai CC, Ting LL, Hsu MM. Quality of life in patients of nasopharyngeal carcinoma: validation of the Taiwan Chinese version of the EORTC QLQ-C30 and the EORTC QLQ-H\&N35. Qual Life Res. 2003;12(1):93-8.

10. Toth G, Sakaguchi T, Mikami Y, Hirose H, Tsukuda M. A pilot study of the translation, cultural adaptation and validation of the EORTC head and neck cancer quality of life questionnaire module (QLQ-H\&N35) for use in Japan. Auris Nasus Larynx. 2005;32(2):175-83.

11. Singer S, Wollbrück D, Wulke C, Dietz A, Klemm E, Oeken J, Meister EF, Gudziol H, Bindewald J, Schwarz R. Validation of the EORTC QLQ-C30 and EORTC QLQ-H\&N35 in patients with laryngeal cancer after surgery. Head Neck. 2009;31(1):64-76.

12. Bower WF, Vlantis AC, Chung TM, Cheung SK, Bjordal K, van Hasselt CA. Quality of life in head and neck cancer patients after surgical resection: translation into Cantonese and validation of the EORTC QLQ-H\&N35. Acta Otolaryngol. 2009;129(7):779-85.

13. Nalbadian M, Nikolaidis V, Nikolaou A, Themelis C, Kouloulas A, Vital V. Psychometric properties of the EORTC head and neck-specific quality of life questionnaire in disease-free Greek patients with cancer of pharynx and larynx. Qual Life Res. 2010;19(5):761-8.

14. Yang Z, Meng Q, Luo J, Lu Q, Li X, Li G, Wan C. Development and validation of the simplified Chinese version of EORTC QLQ-H\&N35 for patients with head and neck cancer. Support Care Cancer. 2012;20(7):1555-64.

15. Carrillo JF, Ortiz-Toledo MA, Salido-Noriega Z, Romero-Ventura NB, OchoaCarrillo FJ, Oñate-Ocaña LF. Validation of the Mexican Spanish version of the EORTC QLQ-H\&N35 instrument to measure health-related quality of life in patients with head and neck cancers. Ann Surg Oncol. 2013;20(5):1417-26.

16. Ouattassi N, Benmansour N, ElFakir S, Nejjari C, Alami MN. Translation and validation of EORTC QLQ-H\&N 35 into Moroccan Arabic for ENT head and neck cancer patients in Morocco. Eur Arch Otorhinolaryngol. 2016;273(9): 2727-34.

17. Singer S, Araújo C, Arraras Jl, Baumann I, Boehm A, Brokstad Herlofson B, Castro Silva J, Chie WC, Fisher S, Guntinas-Lichius O, Hammerlid E, Irarrázaval ME, Jensen Hjermstad M, Jensen K, Kiyota N, Licitra L, Nicolatou-Galitis O, Pinto M, Santos M, Schmalz C, Sherman AC, Tomaszewska IM, Verdonck de Leeuw I, Yarom N, Zotti P, Hofmeister D, EORTC Quality of Life and the EORTC Head and Neck Cancer Groups. Measuring quality of life in patients with head and neck cancer: Update of the EORTC QLQ-H\&N Module, Phase III. Head Neck. 2015;37(9):1358-67.

18. Trivic SK, Trivic A, Singer S, Milovanovic J, Stankovic P, Mikic A, Vukasinovic $M$, Djordjevic V, Jotic A, Folic M, Stevanovic D. European Organization for Research and Treatment of Cancer quality of life questionnaire-head and neck module, updated version: preliminary psychometric data from Serbian laryngectomized patients. Head Neck. 2016;38(Suppl 1):E916-24.

19. Polit DF, Yang FM. Measurement and the measurement of change: a primer for the health professions. Philadelphia: Lippincott Williams \& Wilkins; 2015.

20. Davudov MM, Harirchi I, Amiraliyev N, Mehtiyeva E, Mirzajani Z, Amiraliyev K, Rustamli N, Zebardast J, Montazeri A. The Azeri version of European Organization for Research and Treatment of Cancer Core quality of life questionnaire (EORTC QLQ-C30): translation and validation. Asian PaC J Cancer Prev. 2020:21:267-71.

\section{Publisher's Note}

Springer Nature remains neutral with regard to jurisdictional claims in published maps and institutional affiliations. 